Recepción: 18 / 11 / 2016

Aceptación: 17 / 03 / 2017

Ciencias económicas y empresariales

Publicación: 05 / 05 / 2017

\title{
Aplicabilidad de los principios gerenciales para una gestión empresarial exitosa
}

Applicability of management principles for successful business management

Aplicabilidade dos princípios de gestão para uma gestão de negócios bem sucedida

\author{
David L. Loor-Vélez I \\ davidloorvelez@hotmail.com \\ Daniel A. Miranda-Ledesma II \\ daml_197705@hotmail.com
}

Correspondencia: davidloorvelez@hotmail.com

\footnotetext{
${ }^{I}$ Magister en Comercio y Finanzas Internacionales, Ingeniero Industrial, Docente de la Universidad Laica "Eloy Alfaro" de Manabí, Manta, Ecuador.

II Magister en Dirección de Empresas, Economista con Mención en Gestión Empresarial Especialización Finanzas, Contador Público Autorizado, Docente de la Universidad Laica "Eloy Alfaro" de Manabí, Manta, Ecuador.
} 



\section{Resumen}

Los principios gerenciales tienen un impacto en la forma como las organizaciones logran sus objetivos en un mundo complejo, lo que constituye que el gerente debe tomar decisiones marcadas por la incertidumbre, además de confrontarse con paradigmas gerenciales para desarrollar capacidades de éxito. Estas afirmaciones están enmarcadas en las demandas del entorno de una gerencia, que asume retos del cambio en todas las dimensiones. Desde los modelos gerenciales, se incorpora una serie de variables, constructos que lejos de facilitar los retos del cambio, muchas veces lo obstaculizan para lo cual el gerente debe desarrollar habilidades, así como sentido dialogístico de la complejidad para conciliar las contradicciones. Bajo esta perspectiva, se pretende analizar los modelos gerenciales aplicados en una empresa de formación y capacitación, donde se pudo constar que estos modelos consideran las capacidades de la gerencia de aprender y querer actualizarse para lograr cambios organizacionales en la empresa, así como para adquirir competencias genéricas y no sólo de conocimiento sino en el quehacer de las áreas específicas.

Palabras clave: principios gerenciales; paradigmas gerenciales; complejidad; incertidumbre.

\section{Abstract}

Management principles have an impact on how organizations achieve their goals in a complex world, which means that the manager must make decisions marked by uncertainty, in addition to confronting management paradigms to develop successful capabilities. These statements are framed in the demands of a management environment that assumes challenges of change in all dimensions. From the managerial models a series of variables is incorporated, constructs that far from facilitating the challenges of the change, often they obstruct it for which the manager must develop skills as well as a dialogical sense of the complexity to conciliate the contradictions. Under this perspective, we intend to analyze the managerial models applied in a training and training company, where it could be stated that these models consider management's abilities to learn and want to be updated in order to achieve organizational changes in the company, as well as to acquire generic competences, and not only knowledge but in the work of specific areas.

Keywords: managerial principles; management paradigms; complexity; uncertainty. 


\section{Resumo}

Princípios de gestão têm um impacto sobre como as organizações a alcançar seus objetivos em um mundo complexo, o que é que o gestor deve tomar decisões marcadas pela incerteza, bem como paradigmas de gerenciamento de confrontar a desenvolver habilidades para o sucesso. Essas declarações são enquadradas nas demandas de um ambiente de gerenciamento, que assume desafios de mudança em todas as dimensões. A partir dos modelos de gestão, incorpora uma série de variáveis, constrói longe de facilitar os desafios da mudança, muitas vezes tão apertado para que o gestor deve desenvolver habilidades e senso dialógica de complexidade para reconciliar as contradições. Nesta perspectiva, é analisar os modelos de gestão aplicados em uma educação de negócio e de formação, que afirmou que era possível que esses modelos consideram as capacidades de gerenciamento de aprender e deseja atualizar para alcançar mudanças organizacionais na empresa e adquirir competências genérica e não apenas de conhecimento, mas na tarefa de áreas específicas.

Palavras chave: princípios gerenciais; paradigmas gerenciais; complexidade; incerteza.

\section{Introducción}

En los tiempos actuales, las organizaciones, compañías y empresas de bienes y servicios, tratan de destacarse y posicionarse en el mercado que les es inherente, enfrentando un mundo competitivo donde solo se mantienen y sobreviven aquellas que, para satisfacer la demanda, logran interpretar de manera asertiva la actualidad de un mundo global en una compleja red de relaciones que le exige revisión permanente de sus procesos internos y actualización constante de sus sistemas de gestión. Esto, les obliga a estar constantemente revisando y adaptándose a la dinámica y mercado local, regional, nacional y global, considerando una gran cantidad de factores espaciales y temporales con miras a optimizar su funcionamiento y producción con un máximo de su eficacia, eficiencia y efectividad. Igualmente, las empresas han tenido que enfrentar una competencia cada vez más agresiva, los consumidores son más exigentes y complejos, la tecnología cambia con gran rapidez, la liberación de los mercados internacionales y la emergencia de los bloques económicos afectan el quehacer de las organizaciones empresariales. Lo anterior exige una alta disposición a nivel de gerencial y administrativo debido a que todo proceso de cambio o mejora debe ser apoyado por los líderes. 
En este tenor los principios gerenciales están relacionados a los procesos de cambios organizacionales, innovación, competitividad, liderazgo que van de la mano con los ambientes complejos y cambiantes del entorno. En tal sentido se destacan distintas corrientes para identificar la manera como las organizaciones administran y gerencias sus recursos con la finalidad de sobrevivir en el mercado. De tal forma que los principios o modelos de gestión son promovidos como modelos claves que contribuyen a enfrentar con mayores posibilidades de éxitos el entorno en el que operan. Para Bérdard (2012) los efectos resultan contrarios a lo esperado, pues "la propagación de estos principios conduce a la confusión y la gerencia tiende a desechar modelo para tomar otro generando así, un efecto de parálisis por el exceso de información” (p.72) Desde esta perspectiva esta investigación se propone conocer la aplicación de los principios gerenciales en la empresa CPA Consultores ubicada en la provincia de Guayas, dedicada a la formación y capacitación de profesionales.

\section{Modelos gerenciales y/o paradigmas}

A raíz del desmoronamiento a los cuales muchos autores denominan modernidad, se tiene en el campo teórico una serie de corrientes filosófica que aspiran llenar el vacío dejado por la modernidad. En este sentido la necesidad de alcázar un óptimo de desarrollo empresarial se han propuestos el uso de determinados modelos y con ellos principios gerenciales para entender y fortalecer cada uno de los procesos que se desarrollan en una empresa. Estos serán entonces los paradigmas que constituyen elementos claves que aplican las empresas.

Particularmente en el campo gerencial, se observa como autores como Toffler (1992) miran las facetas históricas de la humanidad en etapas pasando por una 1era ola cuyas características son las actividades agrícolas, seguida de una 2da ola con la llegada de inventos, aparición de máquinas y fuentes de energía iniciando así la industrialización y la maximización de ganancias de las organizaciones, pasando por el desarrollo de las tecnologías de la información llamada la 3ra ola cuya características son la sistematización de microcircuitos hasta llegar a la 4ta ola donde se resalta la creatividad, lo cualitativo e intangible, se busca la innovación como requerimiento imprescindible para las organizaciones empresariales. Estas visiones son acompañadas por modelos gerenciales sustentados a partir de estos paradigmas. 
En este devenir proliferaron modelos de gestión especialmente a partir de la década del 60 con los modelos basados en el árbol de decisiones gestión por contingencia, organización matricial, entre otras. En la década de los 70 los modelos gerenciales giraron en torno a la gestión por objetivos, las tres estrategias genéricas de la cadena del valor, organización por redes de trabajo, etc. mientras en la década de los 80 se establecieron principios gerenciales basado en la descentralización diversificación, gestión de calidad, etc. todos estos enfoques tiene en común la visión paradigmática de las variables básica de la administración: tareas, personas, ambiente, tecnología y competitividad.

En este sentido, la idea fundamental para la implementación de un modelo de gerencial y principios de gestión viene determinado con la posibilidad de desarrollar valores organizacionales para la consecución de ventajas competitivas, ya sea en productos, servicios, costo, tecnología. Para Monge y Zhunio (2013) la adopción de un modelo gerencial también trae consigo riesgos para la empresa de permanecer anclada por mucho tiempo en el mismo lugar, ya que el paradigma puede transformase a menor velocidad que lo que se trasforma el entorno cayendo sí en un desfase paradigma realidad, en donde éste último deja de ser útil (p.46).

Ahora bien, estos modelos de gerenciales han sido útiles fundamentalmente para el mejoramiento de las condiciones de las empresas de producción, se trata entonces de indagar si estos modelos también pueden ser aplicados en empresas de servicio especialmente aquellas dedicas a la formación y capacitación de profesionales cuyos objetivos además de la competitividad están también relacionados con la responsabilidad social, reducción de las desigualdades, fortalecimiento de ciudadanía, etc.

\section{Modelos gerenciales y principios gerenciales}

La gerencia de empresas de servicio de formación y capacitación incluyen en sus procesos de gestión los modelos que se ajustan a su organización para el logro de sus objetivos, en tal sentido es importante destacar los modelos empleados en los últimos tiempos basados en el planteamiento teórico señalando las características y principios de cada uno de ellos.

Planificación estratégica: modelo que contribuye a formalizar los procesos administrativos de forma integral, sus principios gerenciales están vinculados al logro de objetivos organizacionales a través estrategias del análisis del entorno, estudio del mercado, la formulación y diseño de las 
estrategias que direccionen a la empresa a la consecución de sus objetivos de negocio (Palacios, 2010)

Calidad total: este modelo promueve los principios gerenciales hacia el logro de la satisfacción del cliente y del mercado a través de un proceso continuo de generación de valor agregado a los productos y/o servicio, a través de estrategias orientado al cliente tanto interno como externo, (Santos, M., Alvárez L, 2008)

Mejoramiento continuo: modelo que tiene como principios gerenciales la implementación de la calidad utilizando estrategias con énfasis de la participación de los miembros de la organización. Trabaja específicamente tres niveles de la calidad: mantenimiento de procesos, mejoramiento de aquellos cuyo diagnóstico así lo definió, la innovación de toda clase y en todos los puntos del proceso productivo. (Lemaitre, 2009)

Justo a tiempo -Just in time: modelo de calidad esencialmente diseñado para los procesos de producción, enfatiza una filosofía de "calidad en la fuente", lo que significa que deben hacerse bien las cosas desde la primera vez con un control adecuado del proceso de alistamiento, Tiene como estrategia principal mejorar los procesos de producción en línea. (Santos, M., Marin, L, 2000).

Reingeniería: modelo propicio para implementar cuando la producción ha bajado, sus principios gerenciales son la agilización de procesos y sus estrategias es el replanteamiento de la organización enfocada en el rediseño radical. (Rafoso, S., Artiles, S, 2011)

Empoderamiento: modelo gerencial basado en un estilo de liderazgo que desarrolle capacidades de autonomía en el desempeño, los principios gerenciales se vinculan a la toma de decisiones sin mediación de alguna autoridad o presión supervisora. (Hernández, 2010)

Subcontratación, tercerización o externalización: modelo de gestión planificado que transfiere ciertas actividades a subcontratista. Su estrategia es realizar asociaciones entre compañía principal y un tercero a quien se le delega proceso que no generan valor agregado al negocio principal. (Cornejo, 2006).

Este pequeño recorrido de los modelos gerenciales y sus principios señala la complejidad con la que se enfrenta la gerencia a la hora de tomar decisiones en cuanto a las estrategias y principios 
que regirán a la organización, más cuando el entorno y el ambiente interno se caracterizan por la complejidad. El gerente entonces, deberá tener una visión amplia con la finalidad de conciliar tanto las amenazas como las fortalezas y será lo que permita que la organización sobreviva. Por lo tanto, no hay modelo mágico, es a través del aprendizaje mutuo, organización-gerencia lo que hace posible la dinámica de una gestión exitosa, puede decirse entonces que es esencial para la empresa la acción de gerenciar con la visión compleja para regular la variedad generada por la competitividad y la globalización

\section{Metodología}

Dentro de la perspectiva adoptada por la investigación, el estudio tiene un enfoque cuantitativo además aspira obtener una descripción detallada de los principios gerenciales aplicados en la empresa CPA consultores, mediante la observación directa del lugar donde se realizará dándole característica de investigación de campo ya que los datos son extraídos directamente de la realidad de la gestión de la empresa.

Para ello, se consideró los principios gerenciales como consecución de objetivos, satisfacción del cliente, innovaciones en la prestación del servicio, agilización de procesos, descentralización en la toma de decisiones. La investigación se desarrolló en la empresa CPA Consultores cuya población está constituida por 35 personas distribuidas entre el personal administrativo y docente. Al calcular la muestra se utilizó la fórmula para poblaciones finitas

$$
n=\frac{\mathrm{N} * \mathrm{Z}^{2} \alpha \mathrm{p} * \mathrm{q}}{\mathrm{d}^{2} *(\mathrm{~N}-1)+\mathrm{Z}^{2} \alpha * p * q}
$$

Donde $\mathrm{N}$ es el tamaño de la población, $\mathrm{p}^{*} \mathrm{q}$ representa la varianza; Z se refiere al valor obtenido mediante niveles de confianza; y d se refiere al máximo error permitido (Sheaffer,Mendenhall, \& Ott, 2006), en la cual, se calculó el tamaño de la muestra con una precisión o error de 5\%, a un nivel de confianza del $95 \%$ y valores p y q de 50\% (máxima varianza), donde se obtuvo a una muestra total de 24 sujetos en muestreo al azar simple. La técnica de recolección de datos fue la entrevista y se diseñó un cuestionario que fue validado por el método del juicio de expertos el cual es el idóneo para el cálculo de la validez de contenido y calculado por índice porcentual cuyo valor es de 0,83 y la confiabilidad del mismo se calculó aplicando el índice Alfa de Cronbach cuyo valor fue de 0,87 . Igualmente se diseñó un instrumento que se le aplicará sólo a la 
gerencia con la finalidad de conocer cuál es el modelo de gestión que la directiva considera se está aplicando en la organización. Es importante señalar, que la junta directiva ejerce las funciones gerenciales siendo un total de 7 personas, por lo cual se aplicará a toda la población

\section{Resultados}

Para analizar los principios gerenciales aplicados en la empresa CPA Consultores se procedió a identificar las características de los datos en procura de aproximarse a las ventajas o limitaciones que puedan presentase en cuanto al modelo de gestión. En la tabla $N^{\circ} 1$ se muestran los resultados parciales sobre la opinión de los sujetos encuestados en relación a los principios gerenciales de la organización.

Por un lado, se observa que el $80 \%$ de los encuestados la misión, visión y objetivos estratégicos de la organización. Sin embargo, el 67\% manifestó no conocer los planes estratégicos y el 79\% no participa en el diseño de dicho plan. Ahora bien, el 54\% opina que la empresa debería evaluar el desempeño de sus trabajadores, mientras que el 67\% considera que la gerencia no logra los objetivos de la organización. En relación a la existencia de organigrama de responsabilidades los encuestados opinaron saber de su existencia, así mismo, el 58\% piensa que la gerencia no propicia la formación de equipos de trabajo.

Por otra parte, el $88 \%$ opinó que la empresa presta un servicio de cálida a sus clientes y el 54\% piensa que los clientes se sienten satisfechos con la calidad en la formación y capacitación que brinda la empresa CPA Consultores. En otro aspecto de la encuesta, el 58\% piensa que la empresa invierte en instrumentos tecnológicos para la prestación del servicio al cliente y desempeño laboral. En cuanto a la toma de decisiones el $88 \%$ considera que las toma la gerencia en concordancia con el $80 \%$ que opina que no participa en los procesos de toma de decisiones, en cuanto a la promoción de la creatividad e innovación el $63 \%$ opina que la gerencia no la promueve. Sin embargo, el $67 \%$ piensa que el clima organizacional promueve el espíritu colaborativo entre el personal. Por otro lado, el 54\% concuerda en que la gerencia estimula la formación entre sus empleados y el $84 \%$ señalo que la empresa tiene algún servicio en outsourcing. 
Tabla $N^{\circ} 1$

Opinión sobre los principios gerencial de CPA Consultores

\begin{tabular}{|c|c|c|c|c|}
\hline Ítems & TD & $\mathrm{D}$ & A & TA \\
\hline Conoce Ud. la visión, misión y los objetivos estratégicos dela empresa & 0,08 & 0,13 & 0,38 & 0,42 \\
\hline Sabe Ud. de los planes estratégicos de la empresa & 0,38 & 0,29 & 0,13 & 0,21 \\
\hline Participa en el diseño del plan estratégico de la empresa & 0,33 & 0,46 & 0,08 & 0,13 \\
\hline La empresa debe evaluar el desempeño de sus trabajadores & 0,25 & 0,21 & 0,33 & 0,21 \\
\hline La gerencia logra los objetivos que se propone la organización & 0,29 & 0,38 & 0,21 & 0,13 \\
\hline La empresa cuenta con un organigrama de responsabilidades & 0,17 & 0,21 & 0,33 & 0,29 \\
\hline La gerencia propicia la formación de equipos de trabajo & 0,33 & 0,25 & 0,25 & 0,17 \\
\hline Los servicio que presta la empresa son de calidad & 0,08 & 0,04 & 0,5 & 0,38 \\
\hline Los clientes están satisfecho con el servicio que presta la empresa & 0,25 & 0,21 & 0,29 & 0,25 \\
\hline $\begin{array}{l}\text { La empresa invierte en instrumentos tecnológicos para la prestación } \\
\text { de servicio y desempeño laboral }\end{array}$ & 0,21 & 0,21 & 0,25 & 0,33 \\
\hline Las decisiones de la empresa la toma la gerencia & 0,13 & 0,04 & 0,33 & 0,5 \\
\hline $\begin{array}{l}\text { La gerencia de la empresa promueve la creatividad y la innovación en } \\
\text { el personal }\end{array}$ & 0,25 & 0,38 & 0,21 & 0,17 \\
\hline $\begin{array}{l}\text { El clima en la organizacional propicia el espíritu colaborativo entre su } \\
\text { personal }\end{array}$ & 0,17 & 0,17 & 0,42 & 0,25 \\
\hline La gerencia estimula la formación de su empleados & 0,21 & 0,25 & 0,29 & 0,25 \\
\hline Participa en la toma de decisión de la empresa & 0,38 & 0,42 & 0,13 & 0,08 \\
\hline La empresa tiene algún servicio en outsourcing & 0,08 & 0,08 & 0,63 & 0,21 \\
\hline
\end{tabular}

Fuente: Elaboración propia

En relación a la opinión de la gerencia sobre el modelo gerencial aplicados en la empresa el $60 \%$ de los encuestados manifestó conocer los modelos gerenciales más importe, en relación al conocimiento sobre el tipo modelo gerencial el $80 \%$ señala que conoce el modelo de planificación estratégica, el 100\% desconoce el de mejoramiento continuo, el $60 \%$ desconoce el modelo de calidad total, el $60 \%$ señalo conocer el modelo de espíritu emprendedor al igual que el de capital intelectual. En cuanto al modelo de reingeniería el 60\% de los gerentes lo desconoce, mientras que el just in time manifestó conocerlo, mientras que el $60 \%$ desconoce el modelo de empoderamiento.

Ahora bien en cuanto al modelo y/o principio que la gerencia considera que aplica en la empresa (ver Tabla $\mathrm{N}^{\circ}$ 2) lo más resaltante es que el $60 \%$ de los gerentes opinó que aplican el de planificación estratégica, mientras que el $60 \%$ piensa que aplican el Just in time. La gerencia en un $60 \%$ opina que los resultados de la aplicación de los modelos y/o principios gerenciales 
aplicados son favorecedores a la empresa y el $80 \%$ opina que podrían aplicar otros modelos y/o principios gerenciales.

Tabla No2

Opinión de la gerencia sobre los principios gerenciales

\begin{tabular}{|c|c|c|c|c|}
\hline Ítems & TD & $\mathrm{D}$ & A & TA \\
\hline $\begin{array}{l}\text { Conoce Ud. los modelo/o principios de gestión más } \\
\text { importante }\end{array}$ & 0,2 & 0,2 & 0,4 & 0,2 \\
\hline \multicolumn{5}{|l|}{$\begin{array}{l}\text { De modelo/o principios gerenciales que se nombran a } \\
\text { continuación cuál conoce Ud.? }\end{array}$} \\
\hline Planificación estratégica & 0 & 0,2 & 0,2 & 0,6 \\
\hline Mejoramiento continúo & 1 & 0 & 0 & 0 \\
\hline Calidad total & 0,4 & 0,2 & 0,2 & 0,2 \\
\hline Espíritu emprendedor & 0,2 & 0,2 & 0,4 & 0,2 \\
\hline Capital intelectual & 0 & 0,4 & 0,2 & 0,4 \\
\hline Reingeniería & 0,2 & 0,4 & 0,2 & 0,2 \\
\hline Just in time & 0,2 & 0 & 0,4 & 0,4 \\
\hline Empoderamiento & 0,6 & 0 & 0 & 0,4 \\
\hline \multicolumn{5}{|l|}{$\begin{array}{l}\text { Del modelo/o principios señalado cuál cree Ud. que se aplica } \\
\text { en la empresa CPA Consultores }\end{array}$} \\
\hline Planificación estratégica & 0,2 & 0,2 & 0,4 & 0,2 \\
\hline Mejoramiento continúo & 0,6 & 0 & 0,4 & 0 \\
\hline Calidad total & 0,8 & 0,2 & 0 & 0 \\
\hline Espíritu emprendedor & 0,6 & 0,2 & 0,2 & 0 \\
\hline Capital intelectual & 0,8 & 0,2 & 0 & 0 \\
\hline Reingeniería & 0,6 & 0,2 & 0 & 0,2 \\
\hline Just in time & 0 & 0 & 0,4 & 0,6 \\
\hline Empoderamiento & 0,2 & 0 & 0,4 & 0,4 \\
\hline $\begin{array}{l}\text { Cómo califica Ud. los resultados obtenido de la aplicación del } \\
\text { modelo/o principios gerencial en la organización }\end{array}$ & 0 & 0 & 0,4 & 0,6 \\
\hline $\begin{array}{l}\text { Ha pensado la gerencia aplicar otro modelo/o principios } \\
\text { gerencial }\end{array}$ & 0,2 & 0 & 0,4 & 0,4 \\
\hline
\end{tabular}

Fuente: Elaboración propia

\section{Discusión}

En la última década, la industria en general ha experimentado diversos cambios organizacionales y de gestión, debido a que los mercados son cada vez más competitivos y globalizados, por lo mismo, mantener la calidad en el producto o servicio es sumamente importante y es un factor estratégico de éxito. Los costos asociados a los problemas de calidad pueden llevar no sólo a perder ventas, sino a perder la credibilidad y confianza del mercado. En este orden de ideas, los resultados sobre los modelos y/o principios aplicados en CPA Consultores indican por una parte 
un estilo gerencial basado en organización centralizada y piramidal, desde la perspectiva del personal.

Para esta investigación se establecieron las categorías de análisis del estilo gerencias asociadas a las variables de los modelos y/o principios estudiados como son el logro de objetivos organizacionales, satisfacción del cliente tanto interno como externo, innovaciones en la prestación del servicio, agilización de procesos, descentralización en la toma de decisiones. Ahora bien de acuerdo a la opinión del personal la gerencia no tiene una clara política en cuanto a los objetivos organizacionales lo que podría estar influyendo en la actitud de los empleados hacia el logro de objetivos, la toma de decisiones siguen estando en el nivel de la gerencia con poca participación de sus trabajadores, elementos claves de la gerencia moderna. Por otro lado, los modelos mentales de la gerencia siguen marcado la diferencia en las estrategias diseñadas sobre la creatividad e innovación del personal, la cual se manifiesta en la poca capacidad de innovación en el servicio, en los procesos internos, etc. En tanto, no existe la motivación a los trabajadores y por tanto la relación entre calidad, innovación y satisfacción personal es poca. La motivación puede ser estimulada por la participación ya que se aprovecha el potencial creativo del personal. Otro elemento considerado es el sistema de recompensa, para la cual habrá que diseñar estrategia para mejorar los niveles de satisfacción del cliente interno

Sin embargo, desde la perspectiva de la gerencia el modelo y/o principios gerenciales que se aplica en la organización es la planificación estratégica, modelo más conocido y más fácil de relacionar con este tipo de negocio al igual que los principios del Just in time, y que según manifestaron los gerentes estos principios le han dado resultados a la organización. Por ello, se considera que las capacidades de la gerencia de aprender y querer actualizarse son un buen síntoma para lograr cambios organizacionales en la empresa, así como para adquirir competencias genéricas y no sólo de conocimiento sino en el quehacer de las áreas específicas

\section{Conclusiones}

Luego de realizar la investigación sobre los modelos y principios gerenciales aplicados en la empresa CPA Consultores son las más conocidos y de fáciles aplicaciones como lo son la planificación estratégica y Just-time, con un marcado estilo centralizado. Puesto que la gerencia es una actividad que requiere capacidades, habilidades y competencias, además, se concibe al 
gerente como una persona que se desenvuelve en ese contexto complejo y se desarrolla y evoluciona al mismo tiempo, más, sin embargo, el gerente debe, con visión compleja, establecer las interacciones que generen en la organización la consecución de sus objetivos. En otras palabras, los usos proactivos de los elementos aquí señalados añaden valor a los procesos de cambio organizacional el cual gire alrededor de la innovación, el liderazgo y la competitividad, así como sentido dialogístico de la complejidad para conciliar las contradicciones.

\section{Referencias Bibliográficas}

Bérdard, R. (2012). Los fundamentos del pensamiento y las prácticas administrativas. ADMINISTER (3), 68-88.

Cornejo, V. (2006). Del empowerment al outsourcing como estrategia de crecimiento. Obtenido de http://dx.doi.org/10.21503/Ciencia y Desarrollo.2006. v 7.03

Hernández, M. (2010). Empoderamiento institucional y sistemas de información gerencial. El caso de las empresas de economía solidaria en la subregión del Sinú medio, Departamento de Córdoba. Journal of Engineering and Education, 6(10-11), 32-37. Obtenido de http://hdl.handle.net/123456789/5260

Lemaitre, M. (2009). Nuevos enfoques sobre aseguramiento de la calidad en un contexto de cambios. Calidad en la Educación, 31, 166-189.

Monge, E., Zhunio, B . (2013). Aplicación de modelos gerenciales en los establecimientos de alimentos y bebidas del centro histórico de Quito. RICIT(5), 40 - 68.

Palacios, L. (2010). Dirección estratégica. México: ECOE. Ediciones.

Poter, M. (1980). Estrategia Competitiva. Rio de Janeiro, Brasil: Editora Campus Ltda.

Rafoso, S., Artiles, S. (2011). Reingeniería de procesos: conceptos, enfoques y nuevas aplicaciones. Ciencias de la Informática, 42(3), 29-37.

Santos, M., Alvárez L. (2008). Efectos de la Gestión de Calidad Total en la transformación en la Innovación Tecnológica y Administrativa. Obtenido de https://doi.org/10.1016/S11385758(08)70068-9 
Santos, M., Marin, L. (2000). Las técnicas justo a tiempo y su repercusión en los sistemas de producción. Economía Industrial (331), 34-41.

Sheaffer, R., Mendenhall, W. \& Ott, L. (2006). Elementos de muestreo. . Madrid, España: Editorial Paraninfo.

Toffler, A. (1992). La tercera ola. México: Edivisión. 